\title{
Optical bistability in InSb at room temperature with two-photon excitation
}

\author{
A. K. Kar, J. G. H. Mathew, S. D. Smith, B. Davis, ${ }^{\text {a) }}$ and W. Prett|') \\ Physics Department, Heriot-Watt University, Edinburgh, Scotland, United Kingdom
}

(Received 25 October 1982; accepted for publication 7 December 1982)

We report the observation of optical bistability in an InSb resonator at room temperature. This effect and fringe shifts were caused by nonlinear refraction induced by two-photon absorption of radiation from a single longitudinal mode injection-locked pulsed $\mathrm{CO}_{2}$ laser operating at 9.6-10.6 $\mu \mathrm{m}$. Intensities as low as $100 \mathrm{~kW} / \mathrm{cm}^{2}$ were found to be sufficient to tune the $250-\mu \mathrm{m}$-thick cavity through a fringe maximum. From our results we deduce a value of $\chi^{(3)}$ of the order of $10^{-4} \mathrm{esu}$ over the range of intensities investigated.

PACS numbers: $42.65 . \mathrm{Gv}, 85.60 . \mathrm{Me}, 42.60 . \mathrm{Fc}$

The observation of optical bistability in semiconductor devices at room temperature is of great relevance to the practical application possibilities of all optical logic and signal processing elements. Previous observations at infrared wavelengths in narrow band-gap semiconductors have either been performed at cryogenic temperatures ${ }^{1,2}$ in GaAs and InSb or at high input intensities in Te. ${ }^{3}$ Recently, roomtemperature optical bistability has been observed in a GaAsGaAlAs superlattice etalon. ${ }^{4}$ We report here the first observation of optical bistability in InSb at room temperature at both 9.6 and $10.6 \mu \mathrm{m}$ and also the first use of two-photon absorption as the associated free-carrier generation process leading to a large and potentially useful refractive nonlinearity.

We present calculations to predict the magnitude of the effects and we show that up to $10^{17} \mathrm{~cm}^{-3}$ free carriers can be generated in our sample with relatively modest intensities, i.e., a few $\mathrm{kW} / \mathrm{cm}^{2}$. The two-photon absorption process itself has been described elsewhere ${ }^{5}$ and these results are used in our estimates. According to Miller et al. ${ }^{6}$ at an operating frequency around $75 \%$ of that of the energy gap, contributions from both free-carrier plasma and band-gap resonant saturation of conduction states will combine to form the total induced nonlinear refraction in approximately equal magnitude. To estimate the order of magnitude we first calculate the plasma effect.

The density of generated electron-hole pairs $(\Delta N)$ within the sample is determined by the rates of generation, recombination, and diffusion. ${ }^{7}$ If, however, as is usually the case, the diffusion length is much less than the reciprocal of the effective absorption coefficient, then diffusion can be neglected:

$$
\frac{d(\Delta N)}{d t}=\frac{\beta I^{2}}{2 \hbar \omega}-\frac{1}{\tau_{R}}\left(1+\frac{\Delta N}{N}\right)^{2} \Delta N,
$$

where $N$ is the unilluminated carrier density, $\beta$ the two-photon absorption coefficient, $\tau_{R}$ the low excitation electronhole lifetime, and $\hbar \omega$ the photon energy; the factor 2 appears because two photons are absorbed for each transition. $I$ is the incident intensity.

The effect on the refractive index of the sample due to

\footnotetext{
${ }^{a}$ Present address: NPB Technologies Inc., Quebec, Canada.

${ }^{b}$ Present address: Institut für Angewandte Physik der Universität Regensburg, W. Germany.
}

generated free carriers is given by ${ }^{8}$

$$
\Delta n_{p}=-e^{2} \Delta N / 2 \epsilon_{0} n_{0} \omega^{2} m_{c},
$$

where $n_{0}$ is the linear refractive index and $m_{c}$ is the effective mass of conduction-band electrons.

The contribution to the refractive index due to bandgap resonant saturation of the conduction-band states is given by ${ }^{9}$

$$
\Delta n_{b}=\frac{8}{3 \sqrt{ } \pi} \frac{\mu}{m_{0}} \frac{m P^{2}}{\hbar^{2}} \frac{1}{k T} J(a) \Delta n_{p},
$$

where $P$ is the momentum matrix element and $m P^{2} / \hbar^{2} \approx 11$ $\mathrm{eV}, \mu$ is the reduced mass, $m_{0}$ is the electron rest mass, and

$$
J(a)=\int_{0}^{\infty} \frac{x^{1 / 2} e^{-x}}{x-a} d x, a=\frac{\mu\left(\hbar \omega-E_{G}\right)}{m_{c} k T}
$$

and $E_{G}$ is the band-gap energy. Combining Eqs. (2) and (3) we obtain for the total contribution to the refractive index $\Delta n=3.44 \Delta n_{p}$ at $10.6 \mu \mathrm{m}$. We can now calculate the intensity required to generate enough free carriers to change the optical length of the nonlinear cavity by $\lambda / 2$. In our calculations we have assumed a steady state solution for the number of free carriers generated.

Two-photon absorption and photoconductivity in InSb has been studied extensively in our laboratory. ${ }^{5}$ The twophoton coefficient $\beta$ and carrier lifetime $\tau_{R}$ are interrelated in the experimental measurements and are expected to be accurate to a factor of 2 . Taking values of $\beta$ and $\tau_{R}$ as $4.8 \mathrm{~cm} /$ MW and $47 \mathrm{~ns}$ at $10.6 \mu \mathrm{m},{ }^{5}$ calculation shows that we should change the optical thickness of the resonator by $\lambda / 2$ with an intensity as low as $48 \mathrm{~kW} / \mathrm{cm}^{2}$ with $0.7 \times 10^{16} \mathrm{~cm}^{-3}$ free carriers being generated at this intensity.

Local heating effects, due to the absorbed laser energy within the crystal, can be estimated from current data. ${ }^{10} \mathrm{We}$ calculate that the energy required to change the optical path length by $\lambda / 2$ through heating is $\sim 800 \mathrm{~mJ}$ with a thermal diffusion time of $\sim 400 \mathrm{~ms}$. This energy is far greater than the energies used in our experiments $(\sim 20 \mathrm{~mJ})$ and so heating effects should be negligible and have the sense of a positive change of index.

An uncoated plane parallel sample of Te-doped $n$-InSb, carrier concentration $\sim 6 \times 10^{15} \mathrm{~cm}^{-3}$, was used for our investigation. The sample was polished to a thickness of 250 $\mu \mathrm{m}$ and parallelism better than $0.25 \mathrm{mrad}$ in the region of interest. The crystal was mounted on a rotatable table, enabling the resonator to be angle tuned to facilitate fringe 

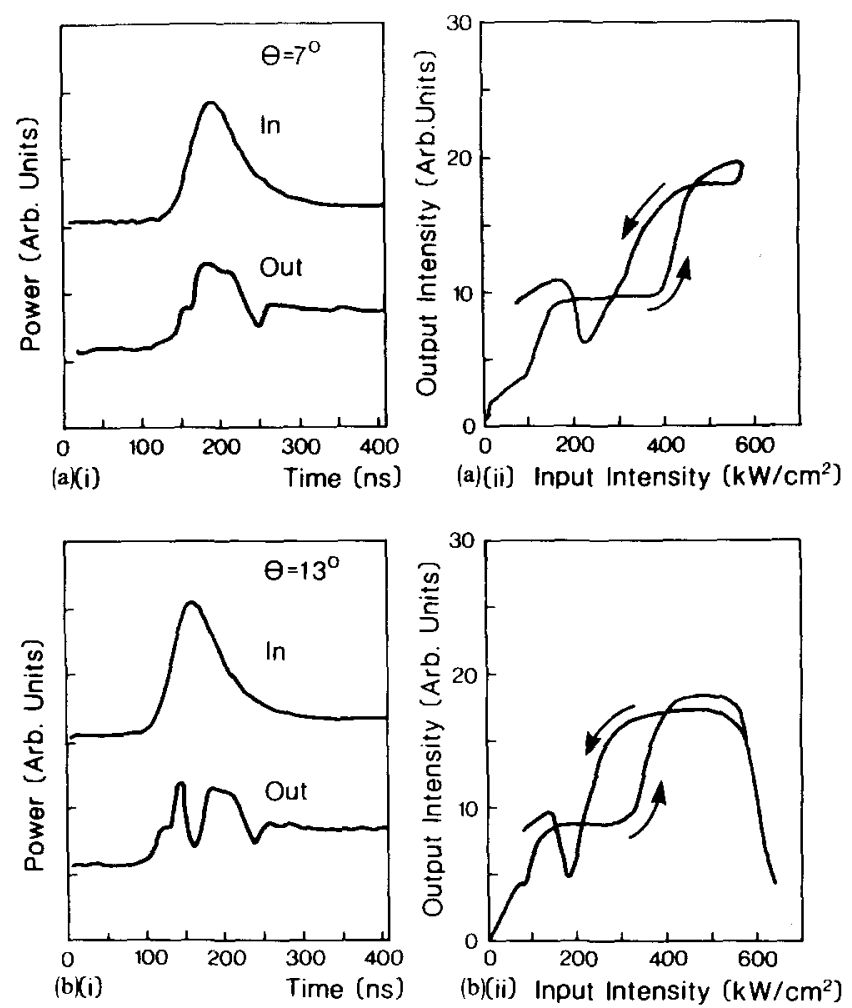

FIG. 1. (i) Variation of transmitted pulse shape with cavity tuning (a) $7^{\circ}$, (b) $13^{\circ}$ for peak incident intensity $\sim 600 \mathrm{~kW} / \mathrm{cm}^{2}$. (ii) Relation between incident and transmitted instantaneous intensities.

observation and to set appropriate detuning for the observation of optical bistability.

Prior to the experiment, rigorous checks were performed with a cw $\mathrm{CO}_{2}$ laser to confirm the presence of interference fringes in the sample. An Edinburgh Instruments $\mathrm{PL} 2 \mathrm{CO}_{2}$ laser was used and the $\mathrm{cw}$ beam was aligned such that both the pulsed and $\mathrm{cw}$ laser beam paths were coincident, so ensuring that measurements were always performed in the same sample portion. Measurements on transmitted power versus angle of rotation confirmed the presence of interference fringes, thus indicating that the sample was indeed parallel in the region of interest. The peak transmission in this low power regime $(\sim 1-2 \mathrm{~W})$ was $\sim 50 \%$ with a coefficient of finesse of 0.4 . Further checks on sample quality using various techniques are in progress and will be reported at a later stage.

An injection-locked pulsed $\mathrm{CO}_{2}$ laser capable of delivering $300 \mathrm{~mJ}$ at $10.6 \mu \mathrm{m}$ with a 150 -ns pulse length in a single longitudinal and transverse mode was used. The low intensity requirement enabled us to use the unfocussed beam with the maximum peak intensity utilized $\sim 600 \mathrm{~kW} / \mathrm{cm}^{2}$, which is considerably lower than the damage threshold. Intensity control was achieved through the use of polyethylene sheets which were individually calibrated prior to use. These were inserted before a spatial filter so that beam quality was maintained.

The radiation transmitted through the crystal was sampled over a 1-mm spot size by means of a variable aperture to the rear of the sample. The beam was first viewed on a Delta Developments beam profiler to ensure that aperturing did not affect the spatial intensity distribution.
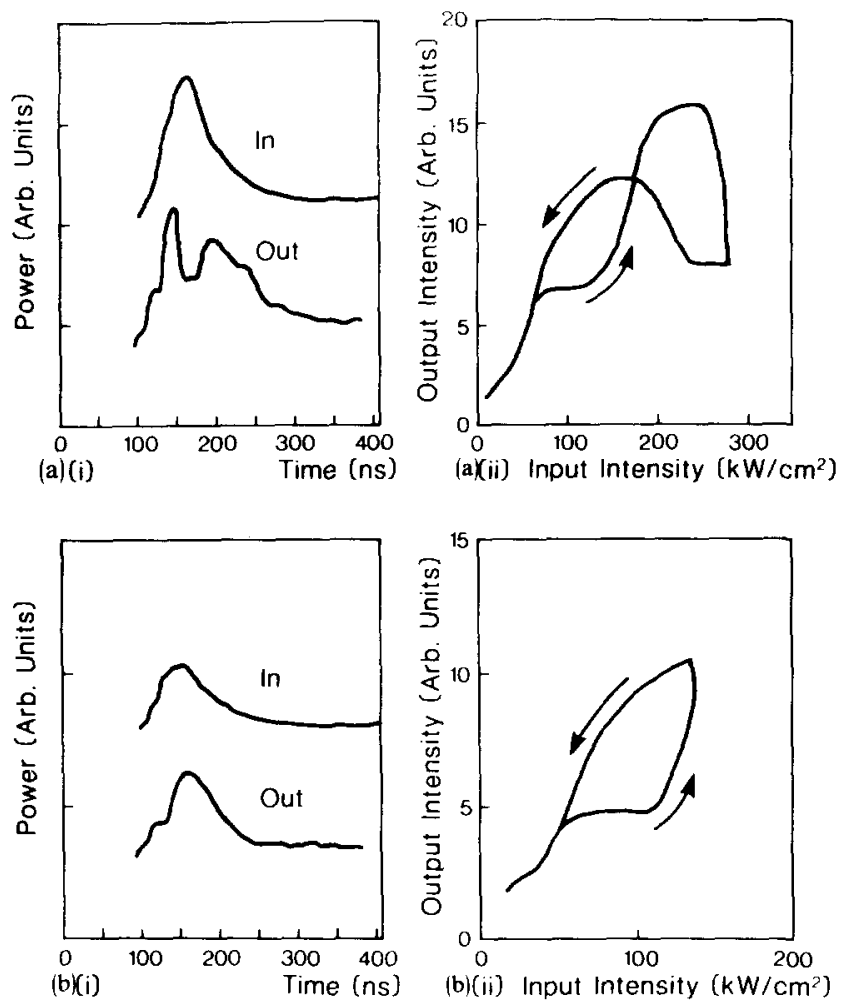

FIG. 2. (i) Variation of transmitted pulse shape with incident intensity (a) $\simeq$ $300 \mathrm{~kW} / \mathrm{cm}^{2}$ (b) $\simeq 200 \mathrm{~kW} / \mathrm{cm}^{2}$. (ii) Relation between instantaneous incident and transmitted intensities.

Both the incident and the transmitted pulses were detected by fast $(\sim 1 \mathrm{~ns})$ photon drag detectors and viewed on a Tektronix 7844 dual beam oscilloscope with a Thomson CSF image intensifier and trace digitizing facility interfaced to a microcomputer for bulk data handling.

Figures 1(i) and 2(i) show the raw data obtained for the sample at different resonator tunings (Fig. 1) and for different peak incident intensities (Fig. 2). These clearly show the self-tuning of the InSb crystal due to laser intensity where each peak in the transmitted pulse corresponds to tuning through a fringe maximum.

For each set of data we have plotted [Figs. 1(ii) and 2(ii)] the corresponding relationship between the input and transmitted instantaneous intensities. These display hysteresis loops associated with optical bistability. These plots have also been obtained dynamically by connecting the incident and transmitted signals to the $x$ and $y$ plates of a Tektronix 7104 with identical results. We also deduce from Fig. 1 that the resulting change in peak position indicates that refractive index is decreasing with intensity whereas if the effect is thermal we would expect an increase. ${ }^{8,9}$ This result, combined with the observed speed of switching, confirms an electronic effect; further experiments up to $20 \mathrm{MW} / \mathrm{cm}^{2}$ also indicate negligible absorptive effects up to $1 \mathrm{MW} / \mathrm{cm}^{2}$.

We can see from these plots that there is a sharp switch up from off-resonance to on-resonance level consistent with a nonlinear cavity with optical feedback. The switch down is slower due to the long lifetime of the carriers. Transient loops are also obvious, consistent with the lifetime of the carriers being of the order of the laser pulse length. The transient effects are smaller at high intensities where the carrier 
lifetime decreases with the increased population.

In conclusion, we have demonstrated that with relatively modest intensities $\left(\sim 100 \mathrm{~kW} / \mathrm{cm}^{2}\right)$ we can induce at room temperature optical tuning in $n$-InSb and also construct optically bistable devices. From our measurements we obtain values of $d n / d I$ as $0.2 \mathrm{~cm}^{2} / \mathrm{MW}$ at $100 \mathrm{~kW} / \mathrm{cm}^{2}$ and 0.12 $\mathrm{cm}^{2} / \mathrm{MW}$ at $500 \mathrm{~kW} / \mathrm{cm}^{2}$. These correspond to a value of $\chi^{(3)}$ of the order of $10^{-4}$ esu over the range of intensities investigated.

This work was supported by SERC and CVD. JGHM acknowledges SERC for a CASE studentship. We are particularly grateful to N. Ross for preparation of the high quality samples used in this study.

'H. M. Gibbs, S. L. McCall, T. N. C. Venkatesan, A. C. Gossard, A. Passner, and W. Wiegmann, Appl. Phys. Lett. 35, 451 (1979).
${ }^{2}$ D. A. B. Miller, S. D. Smith, and A. M. Johnston, Appl. Phys. Lett. 35, 658 (1979).

${ }^{3} \mathrm{G}$. Staupendahl and K. Schindler, Opt. Quantum Electron. 14, 157 (1982). ${ }^{4}$ H. M. Gibbs, S. S. Tarng, J. L. Jewell, D. A. Weinberger, K. Tai, A. C. Gossard, S. L. McCall, A. Passner, and W. Wiegmann, Appl. Phys. Lett. 41, 221 (1982).

'J. Dempsey, J. Smith, G. D. Holah, and A. Miller, Opt. Commun. 26, 265 (1978); C. R. Pidgeon, B. S. Wherrett, A. M. Johnston, and A. Miller, Phys. Rev. Lett. 42, 1785 (1979); A. M. Johnston, C. R. Pidgeon, and J. Dempsey, Phys. Rev. B 22, 625 (1980).

${ }^{6}$ D. A. B. Miller, S. D. Smith, and B. S. Wherrett, Opt. Commun. 35, 221 (1980).

A. F. Gibson, C. B. Hatch, P. N. D. Maggs, D. R. Tilley, and A. C. Walker, J. Phys. C 9, 3259 (1976).

${ }^{8}$ A. Miller, D. A. B. Miller, and S. D. Smith, Adv. Phys. 30, 697 (1981)

${ }^{9}$ D. A. B. Miller, C. T. Seaton, M. E. Prise, and S. D. Smith, Phys. Rev. Lett. 47, 197 (1981).

${ }^{10}$ N. Neuberger, Handbook of Electronic Materials (IFI/Plenum, New York, 1971), Vol 2.

\title{
Optically pumped ring laser oscillation to vibrational levels near dissociation and to the continuum in $\mathrm{Na}_{2}$
}

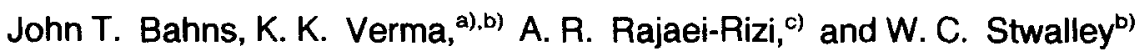 \\ Iowa Laser Facility and Departments of Chemistry and Physics, University of lowa, Iowa City, Iowa 52242
}

(Received 29 October 1982; accepted for publication 1 December 1982)

Unidirectional laser oscillation of $\mathrm{Na}_{2}$ dimers has been observed using $5682-\AA \AA \AA$ single-mode $\mathrm{Kr}^{+}$ laser pumping. This laser action involves not only bound-bound transitions, but also (for the first time) bound-free transitions between two strongly bound electronic states (the $A^{1} \Sigma_{u}^{+}$and $X^{1} \Sigma_{g}^{+}$ states). Twelve bound-bound transitions and two regions of bound-free emission, in the region $7200-8300 \AA$, were observed. Previously reported laser-induced fluorescence results were used to assign both the bound-bound and bound-free transitions to the $A^{1} \Sigma_{u}^{+}-X^{1} \Sigma_{g}^{+}$band system of $\mathrm{Na}_{2}$. The bound-bound transitions are also of particular interest because they all occur to very highly excited vibrational levels within $10 \%$ of dissociation (laser oscillation to lower levels should also be possible but was not examined). The pressure for optimum intensity was found to be $\sim 0.65$ Torr.

PACS numbers: $42.55 . \mathrm{Hq}$

Many new cw optically pumped laser (OPL) sources have been developed in recent years through optical pumping of homonuclear diatomic molecules in the gas phase (eg., $\left.\mathrm{Na}_{2}\right)^{1-5}$. This is due in part to the ease with which these systems may be made to lase. In all of the systems previously demonstrated, discrete output lines are obtained because they operate on bound-bound transitions between strongly bound electronic states. We report here a type of OPL which, in addition to discrete lines, provides relatively broad-band $(\sim 50 \AA)$ laser output. This is to be contrasted with similar broadband emission in excimer systems where the lower electronic state is at most weakly bound.

The distinction between the current system and that of other known excimer systems can be understood by examining the corresponding potential energy curves. The left side

\footnotetext{
a) Present address: 3B-611A, Bell Telephone Laboratory, Holmdel, New Jersey 07733.

${ }^{b}$ Also Department of Physics.

${ }^{c}$ Present address: Department of Chemistry, Drexel University, Philadelphia, Pennsylvania 19104
}

of Fig. 1 (schematic excimer potentials) shows the means by which broadband emission may be obtained in an optically pumped excimer laser. A bound upper state is populated by pumping transitions which occur at larger internuclear dis-
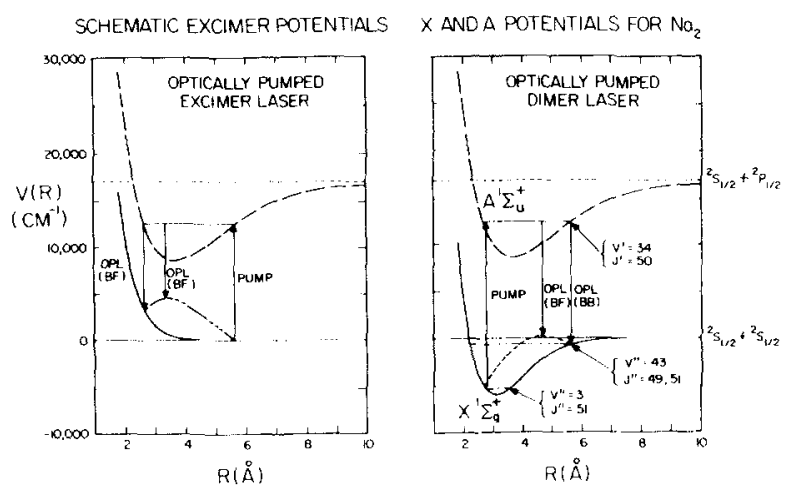

FIG. 1. Potential energy curves for a hypothetical optically pumped excimer laser (left) and for the $A^{1} \Sigma_{4}^{+}-X^{1} \Sigma_{8}^{+} \mathrm{Na}_{2}$ optically pumped laser (right), showing the 5682- $\AA$ pump and resulting bound-bound and boundfree OPL transitions. 